\title{
In-hospital mortality, length of stay, and charges related to pulmonary fibrosis in the U.S. between 1993 and 2008
}

\author{
Daniel Fioret ${ }^{1}$, David Mannino ${ }^{2}$ and Jesse Roman ${ }^{1,3 *}$ \\ ${ }^{1}$ Department of Pharmacology and Toxicology, University of Louisville School of Medicine, Louisville, KY, USA \\ ${ }^{2}$ Department of Medicine, Division of Pulmonary and Critical Care Medicine, University of Kentucky School of Medicine, Lexington, KY, USA \\ ${ }^{3}$ Department of Medicine, Division of Pulmonary, Critical Care, and Sleep Disorders Medicine, University of Louisville School of Medicine and Louisville Robey \\ Rex Veterans Affairs Medical Center, Louisville, KY, USA
}

\begin{abstract}
Purpose: Idiopathic pulmonary fibrosis (IPF) is the prototype of respiratory disorders characterized by lung fibrosis that often lead to respiratory failure and for which there are limited treatments. Data regarding hospital admissions, in-hospital mortality, length of stay (LOS), and charges associated with pulmonary fibrosis are scarce. To bridge this gap in knowledge, we analyzed data available through the Healthcare Cost and Utilization Project (HCUP).

Methods: The HCUPnet Database provides access to national hospitalization data in the U.S. (1993-2008). We analyzed data related to ICD-9-CM codes for post inflammatory pulmonary fibrosis (PPF, ICD-9 code 515) and idiopathic fibrosing alveolitis (IFA, ICD-9 code 516.3) and compared them to those of sarcoidosis (ICD-9 code 135).

Results: IFA discharges declined, especially during 2003-2004, reaching 3,513/year in 2008. PPF and sarcoidosis discharges increased to 30,000 and 7,588/year, respectively. In-hospital mortality decreased for each condition with the largest percent decrease seen in sarcoidosis patients. LOS decreased for PPF and sarcoidosis patients to $\sim 6$ days, but remained between 7.2 and 9.7 days for patients with IFA. Hospitalization charges, however, increased significantly for each condition and reached an estimated 1.48 billion dollars for PPF and IFA combined in 2008.

Conclusions: Although hospitalizations related to IFA decreased slightly, in general, hospitalizations related to pulmonary fibrosis increased since 1993. Meanwhile, LOS and in-hospital mortality decreased, while hospitalization charges increased greatly. These data are important to estimate the financial and other resources required to address pulmonary fibrosis considering that its incidence is increasing and that there are few treatment options.
\end{abstract}

\section{Introduction}

Pulmonary fibrosis affects hundreds of thousands of Americans and many more worldwide [1]. The prototype of this type of illnesses is Idiopathic Pulmonary Fibrosis (IPF), a disorder characterized by progressive scarring of the lungs leading to irreversible distortion of the lung architecture and, ultimately, death [2]. IPF, as the name suggests, has no known cause, but there are several factors associated with a greater risk for disease development and progression including male gender, genetic mutations, smoking, and gastrointestinal reflux disease [2-5]. In other subjects, pulmonary fibrosis develops from known profibrotic stimuli such as allergens, asbestos, and drugs, or is associated with chronic inflammatory conditions such as connective tissue disorders [6,7]. The mortality rate of patients with IPF is $\sim 50 \%$ in 3 years and there are no Federal Drug Administration (FDA) - approved drugs proven to improve survival although 2 drugs appear to slow its progression $[2,8]$. However, mortality rates and treatments differ for other fibrotic lung disorders.

The incidence of IPF appears to be increasing although questions remain as to whether this is related to increased awareness or a true change in disease incidence. This has been influenced by the increased use of high-resolution computed tomography scans [9]. Additionally, the publication by the American Thoracic Society and European Respiratory Society (ATS/ERS) international consensus statement of the diagnosis and treatment guidelines for IPF in 2002 [10] and for idiopathic interstitial pneumonias in 2002 [11] likely increased physician awareness and accurate diagnosis of the disease.

However, more current information about the morbidity and mortality of pulmonary fibrosis is needed. Others have reported a seasonal variation in mortality due to IPF [12] with mortality increasing between 1992 and 2000; the average age- and sex-adjusted mortality rate was 50.8 per $1,000,000$ people [13]. Furthermore, among those with IPF, Blacks are less likely to die from IPF than Whites, whereas Hispanics are more likely to die than Whites [14].

Our analysis focuses on hospital admissions, in-hospital mortality, length of stay, and charges associated with pulmonary fibrosis with sarcoidosis examined for comparison. For this, we utilized a public database containing hospitalization information known as the Healthcare Cost and Utilization Project (HCUP) Public Database.

Correspondence to: Jesse Roman, MD, Pulmonary, Critical Care and Sleep Disorders Medicine, Department of Medicine, University of Louisville, School of Medicine, CTR Building, Room 527, 505 S. Hancock St., Louisville, KY, 40202, USA, Tel: 5058525241; Fax: 5028526233; E-mail: j.roman@louisville.edu

Key words: hospitalization, mortality, pulmonary fibrosis

Received: February 28, 2016; Accepted: March 15, 2016; Published: March 18, 2016 


\section{Materials and methods}

\section{Patient cohort and database analysis}

Data available through HCUP provide access to a family of healthcare databases. These include the Nationwide Inpatient Sample (NIS) which contains inpatient data from a national sample of over 1,000 hospitals in the U.S. as well as the State Inpatient Database (SID) that contains inpatient discharge abstracts from participating states. Data from the databases are provided from 1988 and subsequent years [15]. The NIS is the largest all-payer inpatient care database in the U.S. and contains data from $\sim 8$ million hospital stays yearly and from 1,050 hospitals located in 44 states; approximately a $20 \%$ stratified sample of U.S. community hospitals. The SID contains the inpatient discharge abstracts from participating states and encompasses $\sim 90 \%$ of all U.S. community hospital discharges.

Using the HCUPnet Database, we analyzed data related to ICD-9CM codes for post inflammatory pulmonary fibrosis (PPF, ICD-9 code 515), idiopathic pulmonary fibrosis or idiopathic fibrosing alveolitis (IFA, ICD-9 code 516.3), and sarcoidosis (ICD-9 code 135). We compared different parameters available for each disease (e.g., charges, length of stay, mortality, procedures, diagnoses). We looked at changes over time for the United States (for those states participating in the database) between 1993 and 2008. The classification of co-morbidities was based on the patient's secondary diagnoses.

\section{Results}

\section{Hospital admissions}

Analysis of the HCUPnet Database revealed an increase in number of hospital admissions nationwide between 1993 and 2008 with an upsurge by the turn of the century (Figure 1A). A steady increase in hospital admissions was noted for patients with a principal diagnosis of PPF, doubling in the same time period and reaching close to 30,000

A

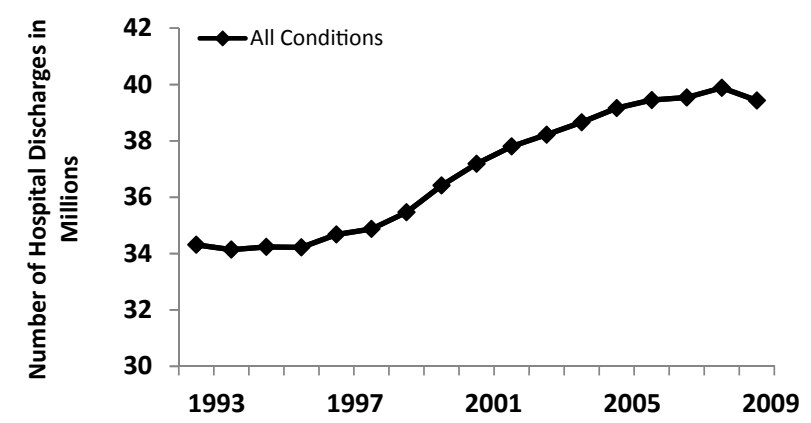

B

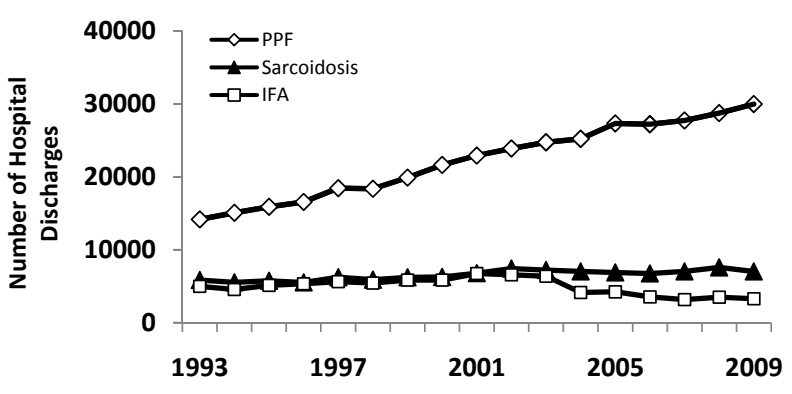

Figure 1. Hospital discharges A) Hospital discharges for all conditions. B) Hospital ,discharges for PPF, IFA, and sarcoidosis. admissions per year in 2008; a $4.9 \%$ increase per year on average (Figure 1B). In contrast, admissions related to IFA were relatively stable, experiencing a decline early in the past decade and reaching 3,513 admissions per year in 2008. For comparison, hospital admissions related to sarcoidosis increased mildly reaching 7,588 admissions in 2008. Interestingly, a significant percentage of patients admitted with a primary diagnosis of PPF, IFA or sarcoidosis also suffered from other important medical conditions (Table 1). The database also included information related to adverse effects of medical drugs ( $\mathrm{E}$ code) showing that up to $16 \%$ of patients hospitalized for IFA suffered from this problem, which was associated with $18.63 \%$ mortality; no information was provided for PPF or sarcoidosis. Table 2 lists the top procedures performed in these patients.

There were many more patients hospitalized with a secondary diagnosis of PPF than those hospitalized with PPF as a primary diagnosis (Figure 2A). Overall, during the 15 year period examined, there was an increase in hospitalizations for patients with PPF where PPF was not the principal diagnosis; from 150,000 in 1993 to 250,000 in 2008. Similar observations were made for IFA, but as before, hospitalizations of IFA patients where IFA was not the principal diagnosis decreased significantly between 2003 and 2004 and reached 15,105 by 2008 (Figure 2B). As observed for PPF, hospitalizations of patients with secondary diagnosis of sarcoidosis increased steadily in the same time period tripling since 1993 (28,638 hospitalizations) to 74,630 admissions by 2008 (Figure 2C).

The above data suggested that patients with diagnosis of PPF, IFA or sarcoidosis are often admitted due to other conditions. Table 3 lists the top primary diagnoses for which patients were admitted. When data were available for an individual diagnosis (e.g., pneumonia), it was evident that subjects with IFA experienced the highest mortality

Table 1. Secondary diagnosis Associated with a principal diagnosis of IFA, PPF, or sarcoidosis. Presented by CCS Code, Secondary Diagnosis, and Principal Diagnosis:\% of Total Discharges (\% Mortality) for IFA, PPF, and sarcoidosis.

\begin{tabular}{|l|l|c|c|c|}
\hline \multirow{2}{*}{$\begin{array}{l}\text { CCS } \\
\text { Code }\end{array}$} & Secondary Diagnosis & \multicolumn{3}{|c|}{$\begin{array}{r}\text { Principal Diagnosis: \% of Total } \\
\text { Discharges (\% Mortality) }\end{array}$} \\
\cline { 3 - 5 } & & IFA & \multicolumn{1}{|c|}{ PPF } & Sarcoidosis \\
\hline 131 & $\begin{array}{l}\text { Respiratory failure, insufficiency, } \\
\text { arrest (adult) }\end{array}$ & $47.8(25.2)$ & $30.1(18.2)$ & $14.5(6.3)$ \\
\hline 98 & Essential hypertension & $44.0(14.1)$ & $42.6(5.8)$ & 39.7 \\
\hline 53 & Hyperlipidemia & $36.3(15.6)$ & $28.2(5.1)$ & 18.3 \\
\hline 127 & $\begin{array}{l}\text { Chronic obstructive pulmonary disease } \\
\text { +bronchiectasis }\end{array}$ & $35.3(9.7)$ & $34.4(6.2)$ & 18.4 \\
\hline 49 & Diabetes mellitus without complication & $31.5(12.1)$ & $24.1(7.6)$ & 23.2 \\
\hline 101 & Coronary atherosclerosis & $31(16.1)$ & $24.5(7.6)$ & -- \\
\hline 257 & Other aftercare & $29.5(17.5)$ & $20.3(13.7)$ & -- \\
\hline 106 & Cardiac dysrhythmias & $28.8(23.2)$ & $21.5(11.2)$ & 13.3 \\
\hline 663 & Screening/history of mental health and & $28.5(14.7)$ & $29.9(4.9)$ & -- \\
\hline 259 & Residual codes, unclassified & $27.8(15.1)$ & $25.5(6.4)$ & 27.2 \\
\hline 103 & Pulmonary heart disease & $27.7(15.8)$ & $17.4(12.8)$ & 13.4 \\
\hline 138 & Esophageal disorders & $26.7(12.7)$ & $23(5.3)$ & \\
\hline 108 & $\begin{array}{l}\text { Congestive heart failure, non- } \\
\text { hypertensive }\end{array}$ & $26.4(12.7)$ & $23.1(11.4)$ & 13.1 \\
\hline 55 & Fluid and electrolyte disorders & $26(25)$ & $20.0(13.5)$ & 17.7 \\
\hline 122 & Pneumonia (not TB/STDs) & $25.2(22.6)$ & $18.7(16.4)$ & 14.6 \\
\hline 244 & $\begin{array}{l}\text { Other injuries/conditions due to } \\
\text { external causes }\end{array}$ & $24.9(17.5)$ & $18.3(11.5)$ & -- \\
\hline 133 & Other lower respiratory disease & $23.1(13.6)$ & $16.5(7.5)$ & 65 \\
\hline 59 & Anemia & $21.7(21.2)$ & $18.2(9.4)$ & 17.6 \\
\hline 2617 & $\begin{array}{l}\text { E codes: adverse effects of medical } \\
\text { drugs }\end{array}$ & $16.1(18.6)$ & -- & -- \\
\hline & & & & \\
\hline & & & & \\
\hline & & & & \\
\hline
\end{tabular}


Table 2. Principal procedure associated with a primary diagnosis of IFA, PPF, or sarcoidosis. Presented by CCS Code, Primary Procedure, and Principal Diagnosis: \% Total Discharges (\% Mortality) for IFA, PPF, and sarcoidosis.

\begin{tabular}{|c|c|c|c|c|}
\hline \multirow[t]{2}{*}{ CCS Code } & \multirow[t]{2}{*}{ Primary Procedure } & \multicolumn{3}{|c|}{ Principal Diagnosis: \% Total Discharges (\% Mortality) } \\
\hline & & IFA & PPF & Sarcoidosis \\
\hline 36 & Lobectomy or Pneumonectomy & 9 & $20.7(1.9)$ & 5.1 \\
\hline 37 & Diagnostic Bronchoscopy and Biopsy of Bronchus & 5.4 & 6.8 & 11.5 \\
\hline 38 & Other Diagnostic Procedures on Lung and Bronchus & 5.6 & $8.8(3.1)$ & 2 \\
\hline 216 & Respiratory Intubation and Mechanical Ventilation & $5.8(67.1)$ & $4.1(49.2)$ & 3.1 \\
\hline 40 & Other Diagnostic Procedures of Respiratory Tract + Mediastinum & 0 & 0.5 & 0.5 \\
\hline
\end{tabular}

A

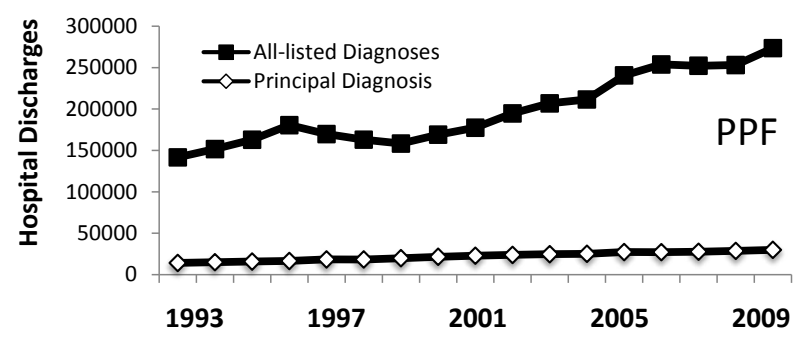

B

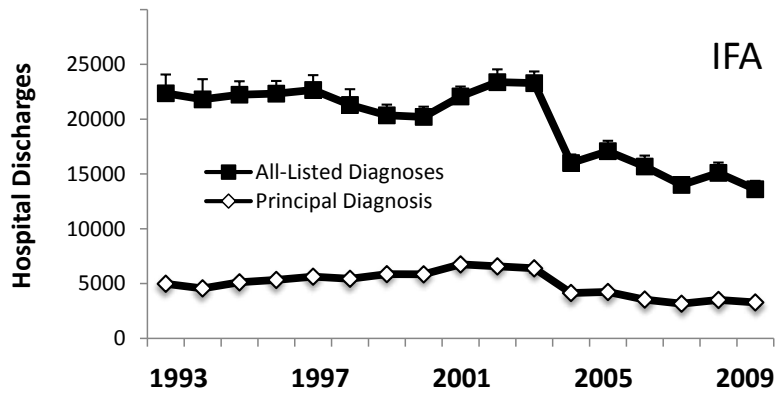

C

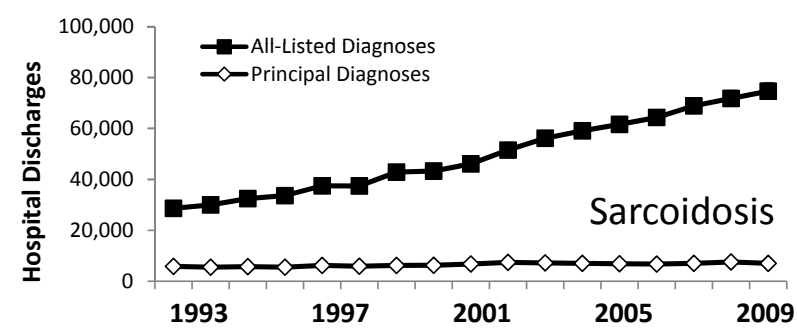

Figure 2. Hospital discharges for PPF, IFA and sarcoidosis as primary versus secondary diagnosis A) Hospital discharges for IFA. B) Hospital discharges for PPF. Hospital discharges for sarcoidosis.

from these conditions followed by PPF. Also, subjects with IFA and PPF showed the highest incidence for critical secondary diagnoses such as respiratory failure.

\section{Length of stay}

Length of stay during hospitalization for all conditions decreased steadily in the mid-1990s and stabilized at 4.6 days by 2008 (Figure 3). Length of stay for PPF, IFA and sarcoidosis was higher (1-3 days) than the average for all conditions. For PPF, length of stay decreased during the mid-1990s and remained relatively stable at 6 days by 2008. On the other hand, length of stay for IFA was the highest of this group starting at 9.7 in 1993, decreasing thereafter, only to increase again in 2007 with an average length of stay of 9.3 days by 2008. For sarcoidosis, length of stay declined from 7.5 days in 1993 to 6.2 days by 2008. Length of stay for the three diagnoses did not appear to differ significantly based on region except for IFA for which the length of stay increased dramatically in 2007 with a return to baseline a year later in the Northwest region (Figure 3B-3D). This did not appear to differ significantly when the data were analyzed for gender. However gender did play a role on percent hospital discharges. For PPF and sarcoidosis, there was a $~ 10$ to $35 \%$, respectively, greater percent of female discharges compared to males (Figure 4B-4C). IFA discharges by gender were not as distinct. We saw more female discharges from 1997-2002 but these values switched in recent years with males comprising the majority of hospital discharges (Figure 4A).

We then determined where the patients were sent after their hospitalization. As depicted in Figure 5A, most patients with PPF ( $65 \%)$, IFA (50\%), and sarcoidosis $(\sim 80 \%)$ demonstrated a 'routine discharge' indicating that they returned home with routine care. However, this number has decreased and by 2008 , over $15 \%$ of patients hospitalized for PPF and IFA were discharged to home health care (Figure 5B). A smaller percentage was discharged to another shortterm hospital (Figure 5C). For PPF and IFA, between 10\% and 15\% of patients were discharged to another institution such as a nursing home or rehabilitation center; this category increased mildly over the time period examined (Figure 5D). As expected, a higher percentage of patients with sarcoidosis were given a 'routine' discharge.

\section{In-hospital mortality}

As presented in Figure 6A, IFA had the highest in-hospital mortality with $14.92 \%$ in-hospital mortality in 2008 , followed by PPF (6.95\%), and sarcoidosis showing the lowest mortality (1.2\%). There has been a small decline in in-hospital mortality for PPF from $9 \%$ in 1993 to $6.95 \%$ in 2008. In-hospital mortality also decreased slightly for IFA from $16.89 \%$ in 1993 to $14.92 \%$ in 2008 . In-hospital mortality for sarcoidosis dropped from $2.3 \%$ in 1993 to $1.2 \%$ in 2008. In general, inhospital mortality is higher for men when compared to women for all three diagnoses (Figure 6B-6D).

\section{Hospitalization charges}

Hospitalization charges have increased for all conditions since 1993 reaching close to $\$ 30,000$ on average (Figure 7A). Similarly, hospitalization charges also increased for IFA, PPF and sarcoidosis. However, while hospitalization charges for PPF (\$15,000 in 1993 to over $\$ 40,000$ in 2008) and sarcoidosis $(\$ 12,563$ in 1993 to over $\$ 37,000$ in 2008) increased at a similar rate, hospitalization charges for IFA increased dramatically in recent years. IFA patients are consistently most costly, especially in the last two years of the analysis when the in-hospital charges for IFA experienced a sharp increase reaching $\$ 81,000$. Differences in hospitalization charges were noted when data 
A

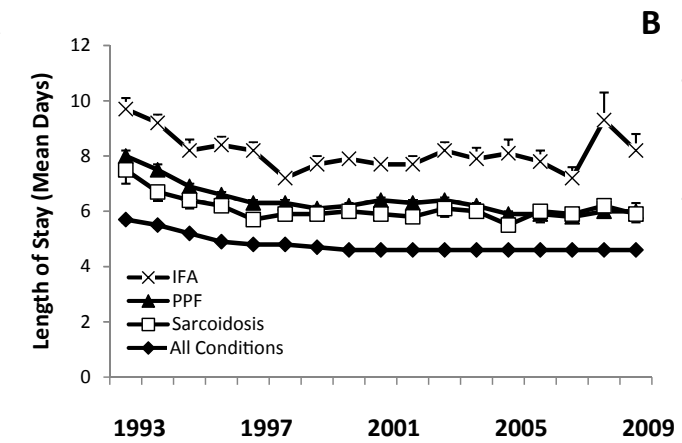

C

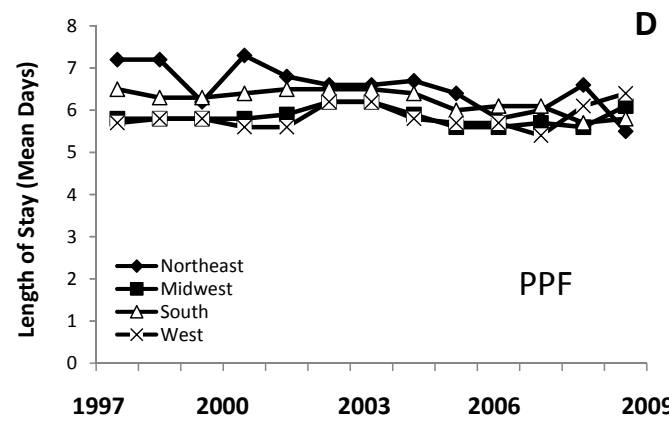

B
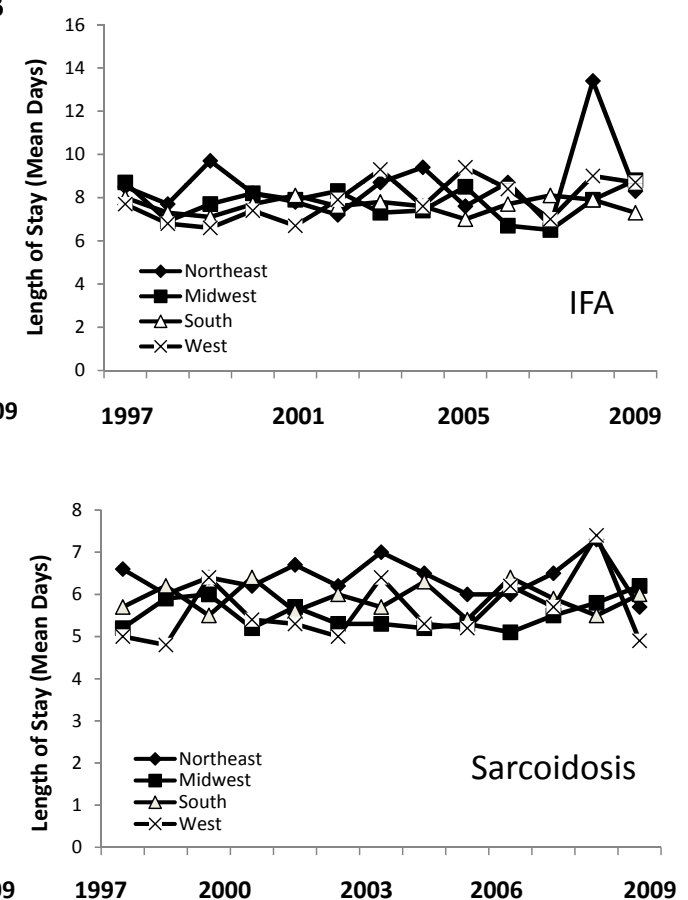

Figure 3. Length of stay A) Length of stay for PPF, IFA, sarcoidosis and all conditions. B) Length of stay for IFA based on region. C) Length of stay for PPF based on region. D) Length of stay for sarcoidosis based on region.

A

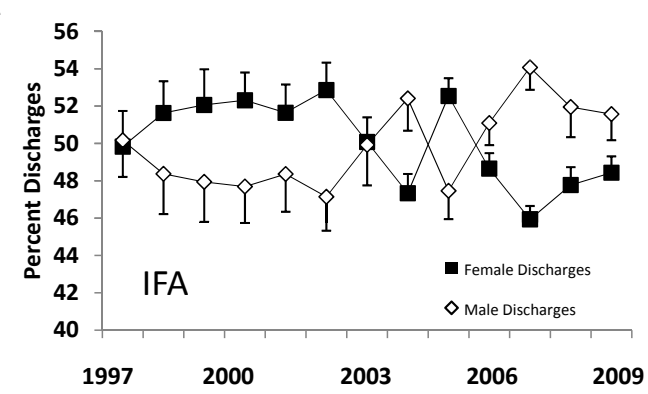

C

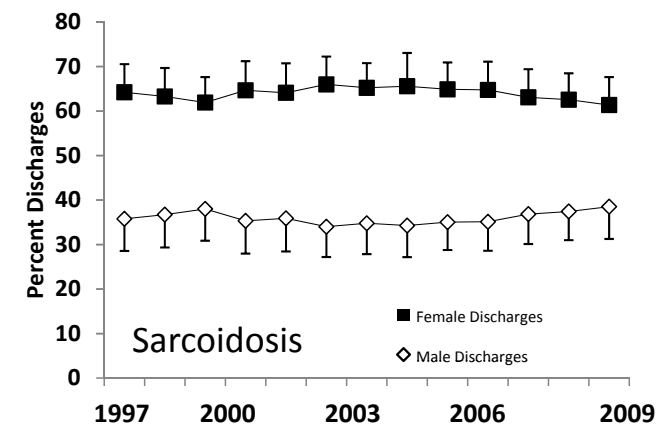

B

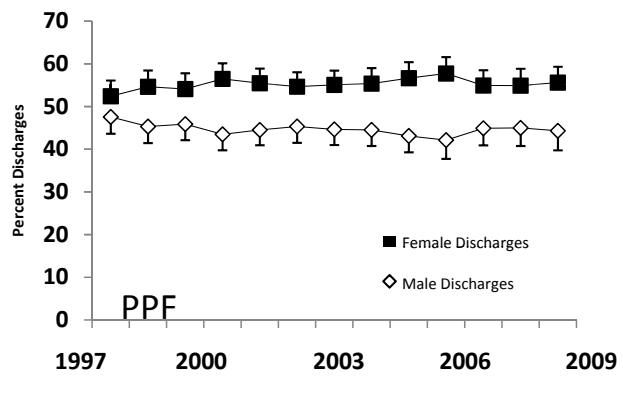

Figure 4. Percent hospitalizations based on gender A) Percent hospitalizations for IFA. B) Percent hospitalizations for PPF. C) Percent hospitalizations for sarcoidosis.

were analyzed by region. For IFA and PPF, hospitalization charges were highest in the West and similar trends were noted for sarcoidosis although data for the latter diagnosis are limited (Figures 7B-D).
Considering that there are significant differences in age of diagnosis for IFA/PPF (more common in elderly subjects) and sarcoidosis (more common in younger subjects) (Figure $8 \mathrm{~A}-\mathrm{C}$ ), we analyzed the data for 

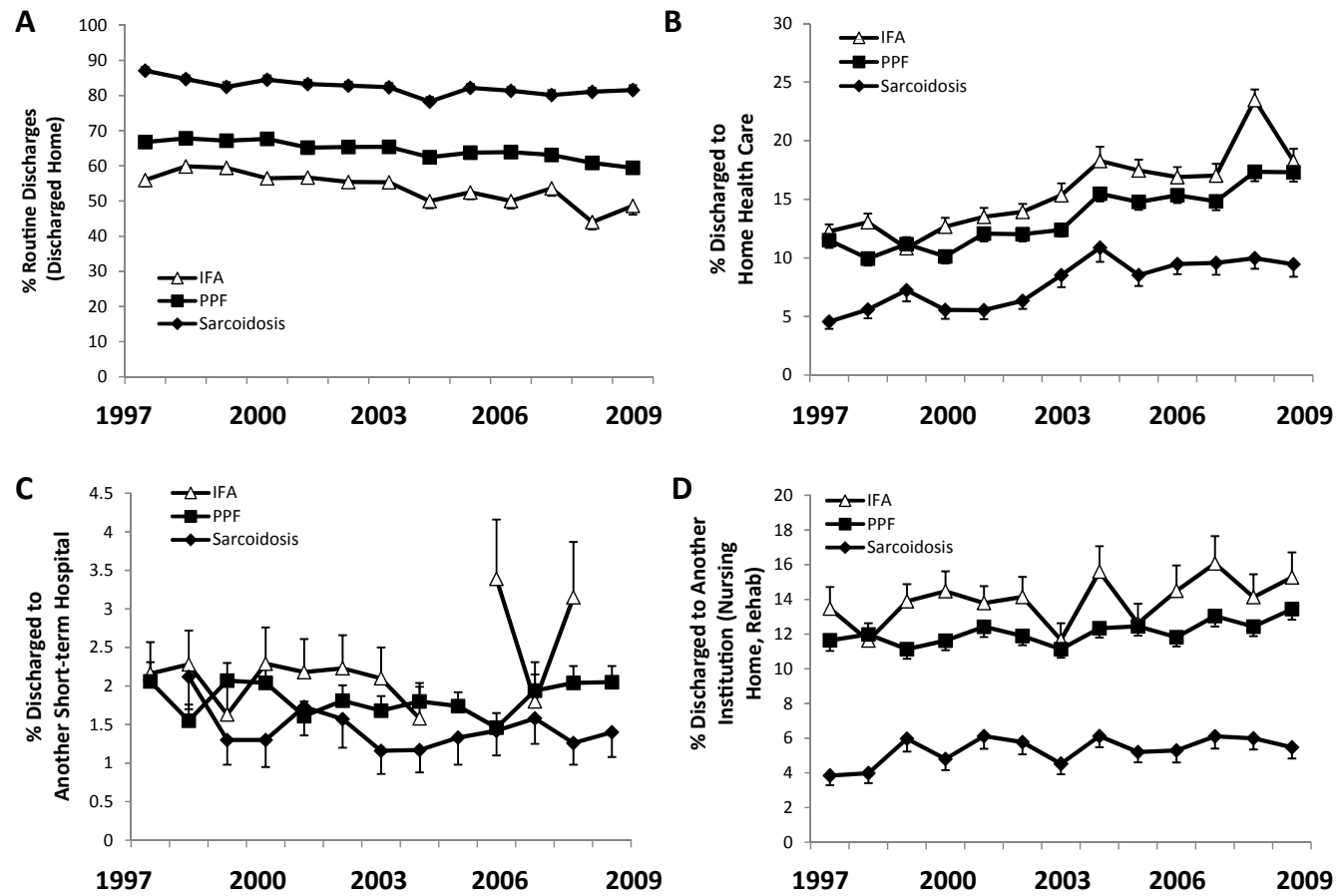

Figure 5. Outcomes after discharge. A) Percent routine discharges for IFA, PPF, and sarcoidosis. B) Percent discharges to Home Health Care. C) Percent discharges to Short-Term Facility D) Percent discharges to another institution.

A

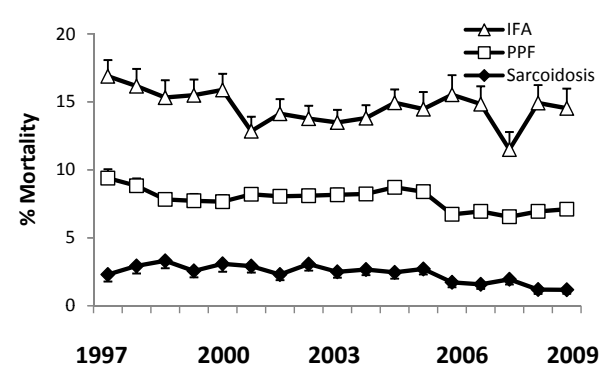

C

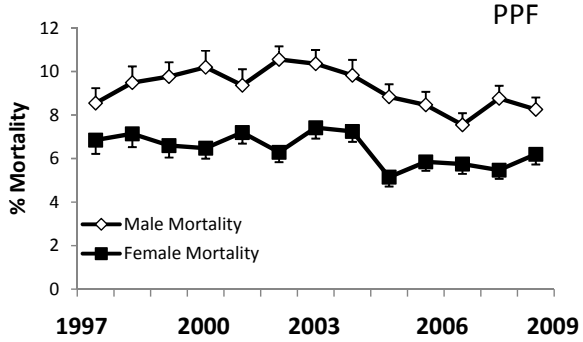

B

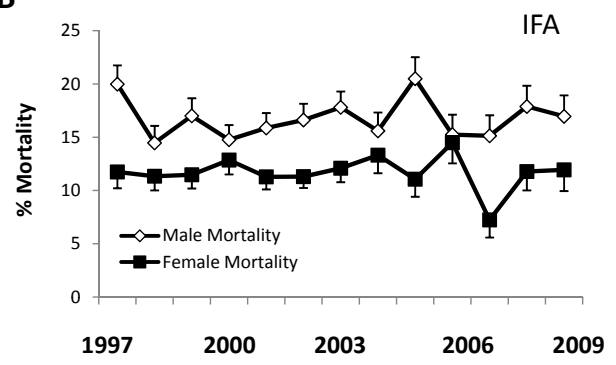

D 5 Sarcoidosis

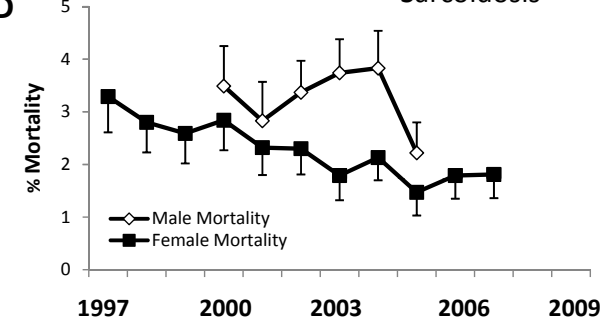

Figure 6. In-hospital mortality A) Percent in-hospital mortality for IFA, PPF, and sarcoidosis. B) Percent in-hospital mortality for IFA based on gender. C) Percent in-hospital mortality for PPF based on gender. D) Percent in-hospital mortality for sarcoidosis based on gender.

each condition based on the percent discharges per age group. IFA and PPF had similar age distribution with the greatest percent of patients being part of the 65-84 age range (Figure $8 \mathrm{~A}-\mathrm{B}$ ). For sarcoidosis, there was a steady increase in the number of 45-64 year old patients while a corresponding decrease was seen in the percent of 18-44 year old patients. We further analyzed these trends by determining the number of discharges per million people by state for 2000 and 2010. However, for each entity, there was no distinct correlation between states with older populations and discharges/million people.

\section{Discussion}

Little is known about pulmonary fibrosis hospitalization rates, inhospital mortality, and cost. We wanted to evaluate this for subjects carrying a diagnosis of IPF, but this remains a difficult task considering the limited databases available and the changes that have occurred regarding IPF diagnosis which limit the accuracy of the classification 

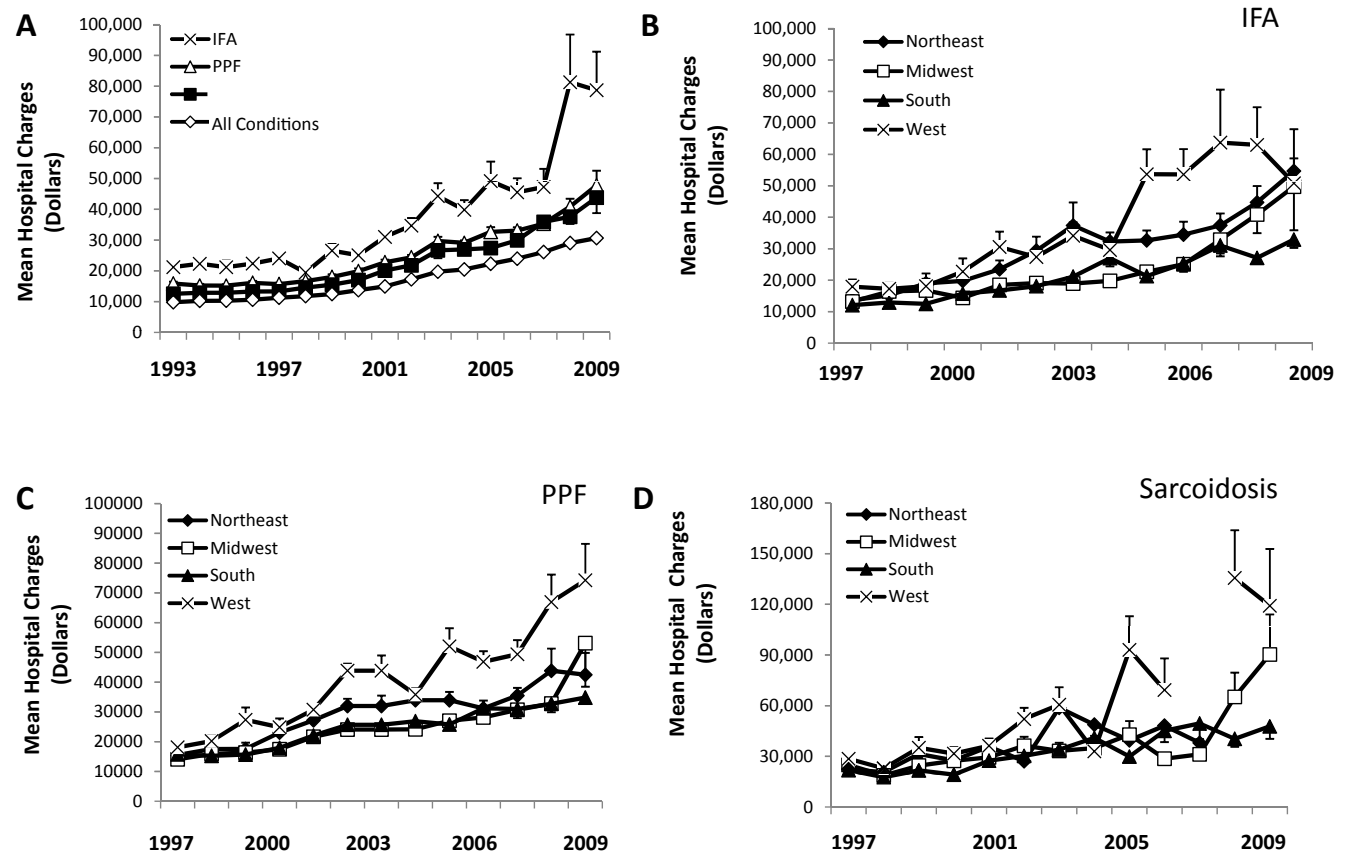

Figure 7. Mean hospital charges A) Mean hospital charges for IFA, PPF, sarcoidosis and all conditions. B) Mean hospital charges for IFA based on U.S. region. C) Mean hospital charges for PPF based on region. D) Mean hospital charges for sarcoidosis based on region.

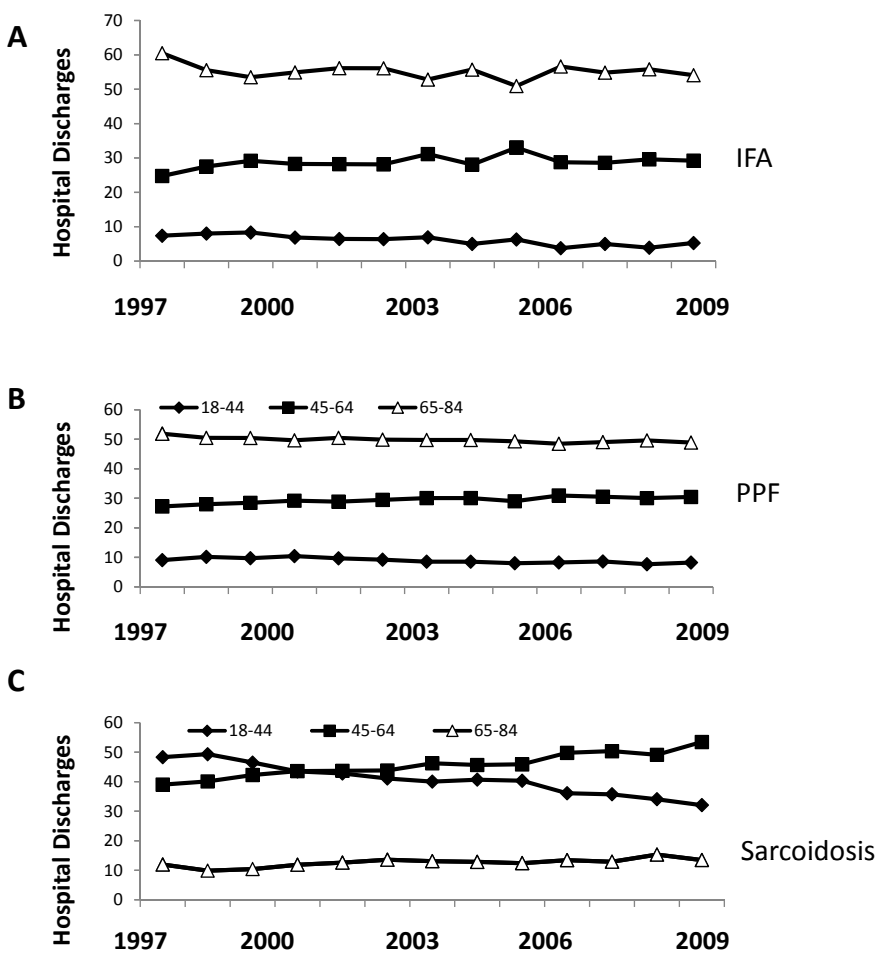

Figure 8. Hospital discharges based on age A) Hospital discharges for IFA based on age. B) Hospital discharges for PPF based on age. C) Hospital discharges for sarcoidosis based on age.

of this disease in hospital documents. Therefore, we evaluated hospitalizations related to the diagnosis of IFA, which is most compatible with the diagnosis of IPF, and PPF, which includes all forms of pulmonary fibrosis independent of the cause. We found that during the 15 year period considered (1993-2008), there was an increase in hospitalizations related to PPF when compared to IFA and sarcoidosis; the latter remaining relatively stable in comparison. Most patients in our cohort were hospitalized with other primary diagnoses suggesting that subjects with these conditions are frequently hospitalized due to comorbidities and/or the need for procedures. Pneumonia and respiratory failure were most frequently listed as the cause of hospitalization. It is likely that some patients with IPF hospitalized with a diagnosis of pneumonia or congestive heart failure suffered instead from an acute exacerbation of IPF [16], but this could not be ascertained. Other co-morbidities included essential hypertension, hyperlipidemia, and diabetes; conditions typically seen in the elderly [17-19]. In our cohort, up to $16 \%$ of patients hospitalized for IFA in 2008 suffered from adverse effects of medical drugs, which was associated with $18.63 \%$ in-hospital mortality and may be explained by the fact that many of these patients are treated with immunosuppressive agents. The number of admissions related to procedures (e.g., diagnostic biopsies, lobectomies) for lung cancer is interesting considering the higher incidence of post-operative complications in these patients [20].

Regarding length of stay, we found a 1-3 day higher length of stay for PPF, IFA and sarcoidosis when compared to all conditions, with IFA having the highest length of stay (7.2 days in 2008). However, length of stay decreased for all three conditions, which is likely due to pressures imposed on physicians for decreasing length of stay. No major differences were noted when gender was analyzed and when U.S. regions were compared.

We then determined where the patients were sent after their hospitalization and noted that by 2008 , over $15 \%$ of patients hospitalized for PPF and IFA were discharged to home health care, which has been increasing steadily over the years, and a smaller percentage was discharged to another short-term hospital. For PPF and IFA, between $10 \%$ and $15 \%$ of patients were discharged to another institution such as a nursing home or rehabilitation center. This percentage has not 
Table 3. Primary diagnoses associated with hospitalization in subjects with the three entities. Presented by CCS Code, Primary Diagnosis, and Secondary Diagnosis: \% of Total Discharges (\% Mortality) for IFA, PPF, and sarcoidosis

\begin{tabular}{|c|c|c|c|c|}
\hline \multirow[t]{2}{*}{ CCS Code } & \multirow[t]{2}{*}{ Primary Diagnosis } & \multicolumn{3}{|c|}{ Secondary Diagnosis: \% of Total Discharges (\% Mortality) } \\
\hline & & IFA & PPF & Sarcoidosis \\
\hline 122 & Pneumonia & $14.6(10.4)$ & $12.8(7.1)$ & $6.5(1.9)$ \\
\hline 131 & Respiratory Failure, Insufficiency, Arrest (adult) & $10.5(31.7)$ & $6.2(21.7)$ & $2.6(11.2)$ \\
\hline 127 & Chronic Obstructive Pulmonary Disease and Bronchiectasis & 8.1 & $9.8(2.2)$ & 3.7 \\
\hline 108 & Congestive Heart Failure, Non-hypertensive & 7 & $8.2(5.4)$ & $4.4(3.2)$ \\
\hline 2 & Septicemia (except in labor) & $5.0(25.2)$ & $4.2(22.7)$ & $3.1(10.3)$ \\
\hline 106 & Cardiac Dysrhythmias & 2.9 & $3.1(3.3)$ & 2.8 \\
\hline 100 & Acute Myocardial Infarction & 2.2 & $2.2(9.3)$ & -- \\
\hline 128 & Asthma & 1.5 & 2 & 3.7 \\
\hline
\end{tabular}

Table 4. Hospitalization charges, LOS, and mortality for patients hospitalized for IFA, PPF, and sarcoidosis (2008).

\begin{tabular}{|l|c|c|c|}
\hline Parameter & IFA & PPF & Sarcoidosis \\
\hline Hospitalizations & 3,513 & 28,732 & 7,588 \\
\hline LOS (days) & 9.3 & 6 & 6.2 \\
\hline Mortality (\%) & 14.92 & 6.95 & 1.2 \\
\hline Charges (\$) & 81,000 & 40,000 & 37,000 \\
\hline Overall charges (Millions of \$) & 285 & 1,149 & 281 \\
\hline *Avg. Charge/Hospital (\$) & 56,797 & $2,29,397$ & 56,039 \\
\hline *Based on www statehealthfacts org total hospital numbers (5,010 for 2008)
\end{tabular}

changed much since 1993, but certainly adds to the financial burden of these disorders.

In-hospital mortality was highest in the IFA group (14.92\%), while PPF had 6.95\% in-hospital mortality; a much lower mortality rate was noted for sarcoidosis (1.2\%). The in-hospital mortality for all groups has declined in the past years. Of note, in-hospital mortality for men was much higher than for women, but the factors responsible for this difference remain unclear.

Finally, we examined hospitalization charges which, as expected, have increased for all conditions since 1993. Hospitalization charges also increased for IFA, PPF and sarcoidosis. However, while hospitalization charges for PPF and sarcoidosis increased steadily, hospitalization charges for IFA increased dramatically in recent years, especially in the last two years of the analysis when the in-hospital charges for IFA experienced a sharp increase reaching $\$ 81,000$. The highest charges were noted for the West region of the U.S. It is unclear how these findings are influenced by hospitalizations related to lung transplantation.

This study has a number of limitations, the most important of which relates to the accuracy of diagnosis. Considering the difficulties inherent in the diagnosis of IPF and related disorders, difficulties inherent in documentation of diagnosis, and the changes made in the classification of idiopathic interstitial pneumonias over the past two decades $[2,10,17,18]$, it is likely that there were misdiagnoses present in the database. Also, some patients may have been counted more than once since there were no unique patient identifiers. However, many of the findings presented were consistent with expectations considering what we know about these disorders as they relate to mortality and gender predilection. Independent of the above, the data suggest that hospitalization rates for pulmonary fibrotic disorders have increased and that this is associated with significant mortality and costs. We estimate that these conditions accounted for 33,513 hospitalizations and 205,293.6 hospitalization days in 2008. About 2,609 of these patients died while in the hospital for $7.8 \%$ in-hospital mortality. The cost of hospitalizations was estimated to be over 1,484,553,000 dollars in 2008 (Table 4).

\section{Conclusions}

Hospitalization rates for pulmonary fibrosis appeared to increase in the past prior two decades. While length of stay and in-hospital mortality decreased, charges increased reaching close to 1.48 billion dollars in 2008. The majority of these patients were admitted because of co-morbidities. Considering the limited options available for the treatment of fibrosis, early identification and treatment of comorbidities might decrease hospitalizations, mortality, and costs for patients with pulmonary fibrosis.

\section{Conflict of Interests}

Dr. Mannino has received honoraria/consulting fees and served on speaker bureaus for GlaxoSmithKline plc, Novartis Pharmaceuticals, Pfizer Inc., Boehringer-Ingelheim, AstraZeneca PLC, Forest Laboratories Inc., Merck, Sunovion, and Amgen. Furthermore, he has received royalties from Up-to-Date and is on the Board of Directors of the COPD Foundation. He has also served as an expert witness on the health effects of tobacco use.

\section{Acknowledgements}

Fioret participated in study design; generation, analysis, and interpretation of data; and manuscript preparation. Mannino participated in study design and interpretation of data as well as manuscript review prior to submission. Roman participated in study design; generation, analysis, and interpretation of data; and manuscript preparation.

\section{References}

1. Raghu G, Weycker D, Edelsberg J, Bradford WZ, Oster G (2006) Incidence and prevalence of idiopathic pulmonary fibrosis. Am J Respir Crit Care Med 174: 810-816. [Crossref]

2. Raghu G, Collard HR, Egan JJ, Martinez FJ, Behr J, et al. (2011) An official ATS/ ERS/JRS/ALAT statement: idiopathic pulmonary fibrosis: evidence-based guidelines for diagnosis and management. Am J Respir Crit Care Med. 183:788-824. [Crossref]

3. Grutters JC, du Bois RM (2005) Genetics of fibrosing lung diseases. Eur Respir J 25: 915-927. [Crossref]

4. Samara KD, Margaritopoulos G, Wells AU, Siafakas NM, Antoniou KM (2011) Smoking and pulmonary fibrosis: novel insights. Pulm Med 2011: 461439. [Crossref]

5. Raghu G, Meyer KC (2012) Silent gastro-oesophageal reflux and microaspiration in IPF: mounting evidence for anti-reflux therapy? Eur Respir J 39: 242-245. [Crossref]

6. Becklake MR, Bagatin E, Neder JA (2007) Asbestos-related diseases of the lungs and pleura: uses, trends and management over the last century. Int J Tuberc Lung Dis 11: 356-369. [Crossref] 
7. Antin-Ozerkis D, Rubinowitz A, Evans J, Homer RJ, Matthay RA (2012) Interstitial lung disease in the connective tissue diseases. Clin Chest Med 33: 123-149. [Crossref]

8. Raghu G; IPFnet (2009) Improving the standard of care for patients with idiopathic pulmonary fibrosis requires participation in clinical trials. Chest 136: 330-333. [Crossref]

9. Lynch DA, Godwin JD, Safrin S, Starko KM, Hormel P, et al. (2005) High-resolution computed tomography in idiopathic pulmonary fibrosis: diagnosis and prognosis. $\mathrm{Am} \mathrm{J}$ Respir Crit Care Med. 172: 488-93. [Crossref]

10. American Thoracic Society (2000) Idiopathic pulmonary fibrosis: diagnosis and treatment. International consensus statement. American Thoracic Society (ATS), and the European Respiratory Society (ERS) Am J Respir Crit Care Med 161: 646-664. [Crossref]

11. American Thoracic Society; European Respiratory Society (2002) American Thoracic Society/European Respiratory Society International Multidisciplinary Consensus Classification of the Idiopathic Interstitial Pneumonias. This joint statement of the American Thoracic Society (ATS), and the European Respiratory Society (ERS) was adopted by the ATS board of directors, June 2001 and by the ERS Executive Committee, June 2001. Am J Respir Crit Care Med 165: 277-304. [Crossref]

12. Olson AL, Swigris JJ, Raghu G, Brown KK (2009) Seasonal variation: mortality from pulmonary fibrosis is greatest in the winter. Chest 136: 16-22. [Crossref]

13. Olson AL, Swigris JJ, Lezotte DC, Norris JM, Wilson CG, et al. (2007) Mortality from pulmonary fibrosis increased in the United States from 1992 to 2003. Am J Respir Crit Care Med 176: 277-284. [Crossref]

14. Swigris JJ, Olson AL, Huie TJ, Fernandez-Perez ER, Solomon J, et al. (2012) Ethnic and racial differences in the presence of idiopathic pulmonary fibrosis at death. Respir Med 106: 588-593. [Crossref]

15. HCUP Overview (2009) Healthcare Cost and Utilization Project (HCUP). Agency for Healthcare Research and Quality, Rockville, MD.

16. Collard HR, Moore BB, Flaherty KR, Brown KK, Idiopathic Pulmonary Fibrosis Clinical Research Network Investigators, et al. (2007) Acute exacerbations of idiopathic pulmonary fibrosis. Am J Respir Crit Care Med. 176: 636-643. [Crossref]

17. Olson AL1, Swigris JJ (2012) Idiopathic pulmonary fibrosis: diagnosis and epidemiology. Clin Chest Med 33: 41-50. [Crossref]

18. Nathan SD, Shlobin OA, Weir N, Ahmad S, Kaldjob JM, et al. (2011) Long-term course and prognosis of idiopathic pulmonary fibrosis in the new millennium. Chest 140: 221-229. [Crossref]

19. Ponnuswamy A, Manikandan R, Sabetpour A, Keeping IM, Finnerty JP (2009) Association between ischaemic heart disease and interstitial lung disease: a casecontrol study. Respir Med 103: 503-507. [Crossref]

20. Sakamoto S, Homma S, Mun M, Fujii T, Kurosaki A, et al. (2011) Acute exacerbation of idiopathic interstitial pneumonia following lung surgery in 3 of 68 consecutive patients: a retrospective study. Intern Med. 50: 77-85. [Crossref]

Copyright: (C2016 Fioret D. This is an open-access article distributed under the terms of the Creative Commons Attribution License, which permits unrestricted use, distribution, and reproduction in any medium, provided the original author and source are credited. 